odyne detection at $10.6, \mu \mathrm{m}$ of the beat frequency between a tunable $\mathrm{Pb}_{0.88} \mathrm{Sn}_{0.12} \mathrm{Te}$ diode laser and a $\mathrm{CO}_{2}$ gas laser," $A p p l$. Phys. Lett., vol. 13, pp. 49-51, July 1968.

[10] J. McElroy, N. McAvoy, H. Richard, T. McGunigal, and G. Schiffner, "Carbon dioxide laser communications systems for near-Earth applications," in Proc. IEEE Int. Conf. Communications, 1970 , pp. 22-7-22-37.

[11] C. Vérié and J. Ayas, " $\mathrm{Cd}_{x} \mathrm{Hg}_{1-x} \mathrm{Te}$ infrared photovoltaic detectors," Appl. Phys. Lett., vol. 10, pp. 241-243, May 1967.

[12] A. Sorrentino, "Infrared detectors, recent development at SAT," presented at the Infrared Symp., Malverne, England, Apr. 1969.

[13] J. Schmitt and E. Stelzer, "Temperature and alloy compositional dependences of energy gap of $\mathrm{Hg}_{1-x} \mathrm{Cd}_{x} \mathrm{Te}, " J$. Appl. Phys., vol. 40, pp. 4865-4869, Nov. 1969.

[14] D. Breitzer, E. W. Sard, B. J. Peyton, and J. McElroy, " $G-R$ noise for Auger band-to-band processes," Infrared Phys., to be published.

[15] B. J. Peyton, "Infrared $10.6 \mu \mathrm{m}$ heterodyne detection in p-type HgCdTe," presented at the Iris Detector Specialty Group Meeting, San Diego, Calif., Mar. 1971.

[16] K. M. van Vliet, "Noise in semiconductors and photocon- ductors," Proc. IRE, vol. 46, pp. 1004-1018, June 1958.

[17] E. N. Figurovskii, P. S. Kireev, A. V. Vanyukov, Yu. V. Evseev, and A. P. Korovin, "Some properties of $p-n$ junctions in $\mathrm{Cd}_{x} \mathrm{Hg}_{1-x}$ Te solid solutions," Sov. Phys.-Semicond., vol. 3, pp. 1572-1573, June 1970 .

[18] J. R. Biard and W. N. Shaunfield, Jr., "A model of the avalanche photodiode," IEEE Trans. Electron Devices, vol. ED-14, pp. 233-238, May 1967.

[19] C. Vérié and M. Sirieix, "Gigahertz cutoff frequency capabilities of CdHgTe photovoltaic detectors at $10.6 \mu \mathrm{m}$," this issue, pp. $180-184$.

[20] I. Melngailis, T. C. Harman, E. D. Hinkley, and W. T. Lindley, "High speed $\mathrm{Hg}_{1-x} \mathrm{Cd}_{x} \mathrm{Te}$ photodiode," presented at the Iris Detector Specialty Meeting, San Diego, Calif., Mar. 1971.

[21] M. I. Skolnik, Introduction to Radar Systems. New York: McGraw-Hill, 1962.

[22] F. P. Pace, R. A. Lange, B. J. Peyton, and F. R. Arams, "Spatially coherent array technology for wide-band infrared heterodyne receivers," this issue, pp. 246-252.

[23] T. McGunigal, J. McElroy, and N. McAvoy, NASA Goddard Space Flight Center, and B. Walker, R. Wong, and J. Cernius, Aerojet General Corp., private communication.

\title{
Wide-Band Laser Communications in Space
}

\author{
DONALD C. FORSTER, FELLOW, IEEE, FRANK E. GOODWIN, MEMBER, IEEE, AND \\ WILLIAM B. BRIDGES, FELLOW, IEEE
}

\begin{abstract}
Candidate wide-bandwidth (1-Gbit/s) satellite laser communications systems are compared on two different bases. First, a comparison is made with projected component technology to establish relative performance between the various approaches based on the fundamental system parameters. From this comparison it appears that the $\mathrm{CO}_{2}(10.6-\mu \mathrm{m})$ system offers a signal-to-noise advantage over the Nd:YAG (1.06- $\mu \mathrm{m})$ or doubled Nd:YAG (0.53$\mu \mathrm{m})$ system for a comparable satellite burden. Second, a comparison is made based upon the concept that launch cost for equivalent systems comprises an optimizing criterion. From this comparison it appears that the launch costs for the $\mathrm{CO}_{2}$ and doubled $\mathrm{Nd}$ :YAG systems can be similar, but the latter is very sensitive to the projected weight of a large lightweight "photon bucket" receiving aperture. In general, the relative deficiency in signal-to-noise ratio for the Nd:YAG system can only be accommodated through adoption of an open-loop pointing system with an accuracy of $1 \mu \mathrm{rad}$ or less, as compared to a closed-loop pointing system with relaxed accuracy for the $\mathrm{CO}_{2}$ system. The most critical technology problem for the $\mathrm{CO}_{2}$ system is that of Doppler compensation. These and other critical technologies for both approaches are listed and discussed.
\end{abstract}

\section{INTRODUCTION}

【1

ASER communications systems in space offer substantial promise in providing heretofore unavailable communications rates $(1 \mathrm{Gbit} / \mathrm{s})$ and, in some situations, lighter weight, more compact systems at lower rates (hundreds of $\mathrm{Mbit} / \mathrm{s}$ ). This paper addresses the status of candidate systems in an attempt to weigh

Manuscript received August 31, 1971; revised September 23, 1971. The authors are with Hughes Research Laboratories, Malibu, Calif. 90265. their relative prospects. The capability of each system is described using available or near-term projected technology. The wideband laser communications missions to be covered will be limited to relay communication between satellites in synchronous orbits or between synchronous and low earth orbits. Operating lifetimes of five years or greater are desired for such relay-type missions, and this requirement influences the discussion to some extent. All laser sources which can be used at present to satisfy the mission objectives lie within the range of 0.5 to $10.6 \mu \mathrm{m}$. Systems within this range also divide naturally into those in the visible and near IR where photoemitting detectors are used and those in the intermediate IR where heterodyne detection is required.

Two methods for comparison of the candidate systems are developed. The first utilizes fundamental parameters in the signal-to-noise relations to provide an estimate of the relative performance to be realized. Certain assumptions about practical parameter choices are made to properly orient the comparison. This method suffers to some extent because it lacks the perspective introduced by engineering and physical limitations, e.g., tolerance versus weight trades, total weights and volumes, costs, etc.

To circumvent these limitations, a second method of comparison is utilized. In this comparison, an approach to an optimized system design is described, and the total burden on the satellite which is incurred by each candidate system is assessed. To allow for a valid comparison, all burdens are interpreted in terms of effective launch wieght, which in turn has a cost equivalence. 


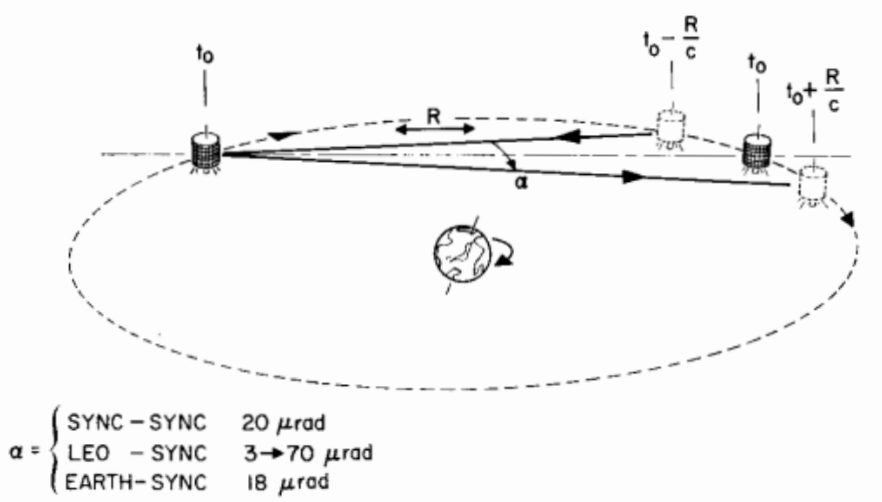

Fig. 1. Two-satellite geometry illustrating the requirement to point-ahead in space for retransmission of data back to the originating satellite. The point-ahead angle $\alpha$ is given for three common satellite situations. The values for low-earth-orbiting (LEO) to synchronous vary over the range given during each orbit of the LEO satellite.

The results of these comparisons serve to provide a total picture including both fundamental considerations and practical design limitations. System definitions are sufficiently detailed to allow identification of the key technology improvements which are required for each candidate approach in order to realize its promise in an operational sense. Since technology improvements of differing natures are required for all approaches, the choice of the best approach must be based upon the judgment of the reader and his confidence in achieving the various increments in performance.

\section{Choice of Model Link}

In order to simplify the discussion to manageable proportions, the particular link (out of all the possible combinations including orbiting terminals, synchronous terminals, and earth terminals) that most stresses the technology will be chosen for consideration. This also turns out to be the link configuration of greatest practical interest, i.e., a wideband (assumed $1 \mathrm{Gbit} / \mathrm{s}$ ) link from a low-earth-orbiting/(LEO) satellite to a synchronous satellite. The utility of this link follows from the observation that very large quantities of data are accummulated by sensors in orbiting satellites, and transmission of this information to the ground is most conveniently accomplished without on-board data storage by a continuous wideband relay through a synchronous satellite.

This link configuration stresses the technology through introduction of the following. 1) The maximum radial component of relative velocity is encountered, which in turn introduces maximum Doppler shifts and timing problems to the systems. 2) Successive acquisition of the orbiting satellite is required each time it appears on the horizon. 3) Tracking coverage which exceeds hemispherical is introduced for the orbiting satellite. 4) Maximum lead or point-ahead angles from the apparent to the real position of the other satellite are introduced. 5) Maximum background radiation from the surface of the earth must be accommodated. This choice does, however, result in the neglect of atmospheric propagation phenomena that would be introduced in a link to a ground terminal.
TABLE I

Candidate Laser Systems

\begin{tabular}{|c|c|c|}
\hline & $\mathrm{Nd} / \mathrm{YAG}$ & $\mathrm{CO}_{2}-\mathrm{N}_{2}-\mathrm{He}$ \\
\hline$\lambda, \ldots$ & $1.06 / 0.53 \mu \mathrm{m}$ & $10.6 \mu \mathrm{m}$ \\
\hline Modulation & PCM-AM or subcarrier & PCM DSSC \\
\hline Detection & Direct & $\begin{array}{l}\text { Heterodyne with } \\
\text { Doppler track }\end{array}$ \\
\hline $\begin{array}{l}\text { Pointing and } \\
\text { tracking }\end{array}$ & $\begin{array}{l}\text { Precise pointing accuracy } \\
\text { with point-ahead } \\
\text { requirement }\end{array}$ & $\begin{array}{l}\text { Nominal pointing } \\
\text { accuracy with } \\
\text { closed- loop track }\end{array}$ \\
\hline
\end{tabular}

a Double sideband suppressed carrier.

The concept of lead or point-ahead angles deserves some elaboration. It may be understood by considering the two orbiting satellites illustrated in Fig. 1. The flight time of a photon over the large distances involved $\left(\approx 4 \times 10^{4} \mathrm{~km}\right)$ is significant. A signal transmitted by one satellite at $t_{0}-R / c$ is received by a second satellite at $t_{0}$ and is immediately retransmitted to the first satellite. Retransmission will have to lead the reception direction by an angle $\alpha$, as shown, to intercept the position of the first satellite at time $t_{0}+R / c$. The magnitudes of the lead angles which are encountered in various link choices are shown in Fig. 1. Note that even "stationary" satellites have point-ahead angles.

\section{Candidate Laser Systems}

A survey of present laser system candidates for wideband space communications converges quickly to two choices upon cursory analysis of parameter requirements. The crucial parameters are the power and efficiency of the laser source. Table I summarizes the general characteristics of the leading candidates at this time.

The Nd:YAG laser operating at $1.06 \mu \mathrm{m}$, or alternatively, doubled to $0.53 \mu \mathrm{m}$, is capable of meeting the average power requirement, although somewhat deficient in efficiency, at present. Modulation formats which have been proposed for these systems are pulse-code modulation-amplitude modulation (PCM-AM) [1] and quadriphase subcarrier modulation [2]. Photoemission followed by electron multiplication is the preferred approach to detection. These Nd:YAG systems in general must employ very narrow transmitter beams (high antenna gain) to provide adequate antenna gain signal-to-noise ratios.

The $\mathrm{CO}_{2}$ laser operating at $10.6 \mu \mathrm{m}$ on any one of its strong vibration-rotation spectral lines ( $P$ or $R$ brunch) is the other viable candidate. The form of modulation which yields the best performance is internal coupling modulation [3]. The output can best be characterized as double-sideband suppressed carrier, and it is compatible with PCM. Since the detectors used at $10.6 \mu \mathrm{m}$ are limited to photoconductors or photovoltaic junctions, heterodyning must be employed to obtain near quantum noise-limited operation. This in turn requires singlefrequency operation and the attendant problems associated with Doppler shift. For this system, beamwidths of the 
order of $100 \mu \mathrm{rad}$ are used; these exceed maximum lead angles to be encountered, and thus the point-ahead problem is avoided.

\section{Comparison on the Basis of Fundamental PARAMETERS}

It is often difficult to arrive at an acceptable basis for comparing systems which employ different basic principles. This is not basically a problem of being unable to assess performance relative to the incurred penalty or burden, but more a problem of interpreting subtle details that restrict the freedom of choice within the system and of assessing practical difficulties and limits in various technology areas. For that reason, two levels of comparison are needed; one which assumes that technology problems will be resolved and attempts to establish the relative merits on a fundamental basis, and a second one which includes the discussion of the technology problems and attempts to identify and document the performance increments that are required. The latter should also provide some insight into the relative difficulties and constraints in implementing the alternative approaches.

This section contains a comparison of the first type, i.e., the fundamental physical parameters are compared to establish some limits and illuminate the problems. The comparison is made on a signal-to-noise basis, even though digital systems with specified bit error rates (BER) and channel capacity are ultimately of interest. Strictly speaking, it is not always possible to relate BER to signal-to-noise ratios, e.g., for direct detection optical PCM. However, an approximate relation sufficiently accurate for this comparison can be obtained, as will be shown later.

The communication signal-to-noise relation is given by

$$
\begin{aligned}
\frac{S}{N} & =\frac{P \eta_{T} \eta_{R}}{h \nu B} \theta_{T}{ }^{2} A_{R}\left(\frac{4 L}{\pi R^{2}}\right) \\
& =\frac{P \eta_{T} \eta_{R}}{h \nu B}\left(\frac{\pi D_{T}}{6 \lambda}\right)^{2} A_{R}\left(\frac{4 L}{\pi R^{2}}\right)
\end{aligned}
$$

where the transmitter beamwidth $\theta_{T}$ is given by

$$
\theta_{T}^{2}=\left(\frac{6 \lambda}{\pi D_{T}}\right)^{2}
$$

the transmitter (laser pulse modulator) efficiency $\eta_{T}$ is defined as

$$
\eta_{T}=\frac{m^{2} \eta_{L} P_{L}}{P_{L}+P_{M}}
$$

and the receiver efficiency $\eta_{R}$ is defined as

$$
\eta_{R}=f \eta_{D} .
$$

The remaining terms are the laser prime power $P_{L}$, the modulator prime power $P_{M}$, the transmitter prime power $P$, where $P=P_{L}+P_{M}$; the modulation index $m$, the laser efficiency $\eta_{L}$, the transmitter aperture diameter $D_{T}$, the receiving aperture area $A_{R}$, the total optical loss through receiver and transmitter $L$, the range $R$, a factor $f$,

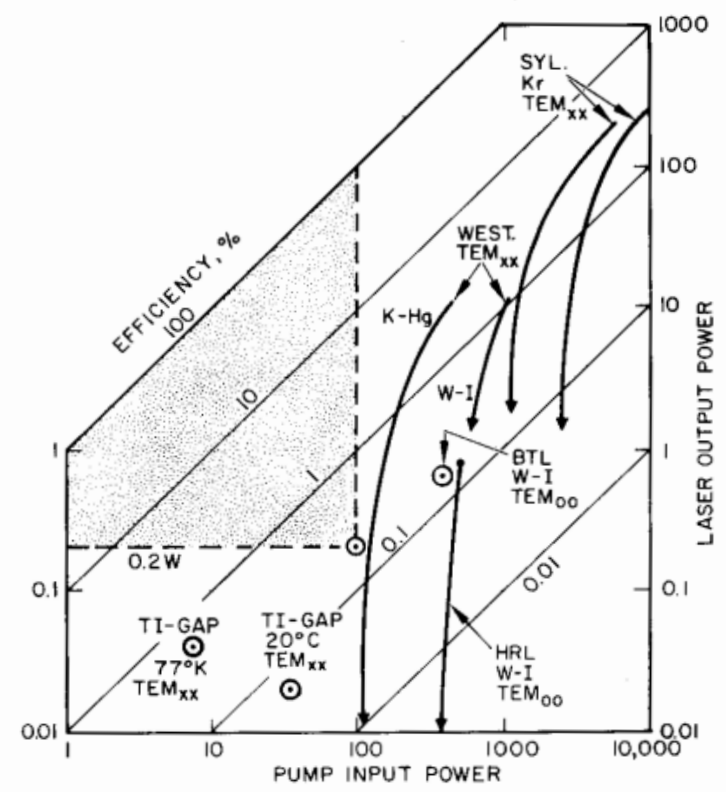

Fig. 2. Summary of Nd:YAG output power versus pump input power from several references: TI-GAP $77^{\circ} \mathrm{K}$ [4]; TI-GAP $20^{\circ} \mathrm{C}$ [5]; WEST. [6]; SYL. [7]; HRL [8]; BTL [9].

less than unity, dependent on the type of detection employed, the detector quantum efficiency $\eta_{D}$, the receiver bandwidth $B$, and $\nu$ and $\lambda$, the operating frequency and wavelength, respectively. The factor of 6 in the transmitter beamwidth expression is derived from the assumption that a departure by a factor of 1.5 from the ideal diffraction limit (relative to an infinite Gaussian illumination function for which $\theta=4 \lambda / \pi D$ ) will be encountered due to truncation, obscuration, optical distortion, etc.

To compare the systems from a signal-to-noise point of view, appropriate values of the parameters must be substituted. Because some technologies are deficient at this time, judgment must be exercised to obtain near-term performance projections. The most critical judgments relate to the performance which can be projected for the $\mathrm{Nd}$ :YAG laser. For this reason it is appropriate to review the state of the Nd:YAG art in more detail. In Fig. 2, a summary of Nd:YAG performance data at $1.06 \mu \mathrm{m}$ from the most appropriate references [4]-[9] is presented. The shaded area represents that performance which is deemed necessary to deploy a practical system; a minimum of $0.2-\mathrm{W}$ output is required to avoid using extremely narrow beams (i.e., less than $1 \mathrm{~s}$ of arc). The $100-\mathrm{W}$ transmitter prime power is the most that is assumed compatible with the classes of satellites under consideration. Lowest order transverse or $\mathrm{TEM}_{00}$ mode operation is desired to achieve near diffraction-limited transmitter optical efficiency. The data labeled as $\mathrm{TEM}_{x z}$ indicate that these are higher order mode lasers, not suitable for the missions considered here. Introduction of mode control methods may cause a degradation in power by as much as one half. Moreover, doubling to $0.53 \mu \mathrm{m}$ will most probably result in an additional degradation of similar magnitude for operational systems, although in principle this can be avoided [10]. The nearest approach to the desired operating range 
IEEE JOURNAL OF QUANTUM ELECTRONICS, FEBRUARY 1972

has been achieved by Lieberman et al. [6] using a potassium-mercury lamp. The nearest mode controlled lasers are those of Evtuhov (labeled HRL) [8] and Chesler and Maydan (labeled BTL) [9]. The light-emitting diode pumped lasers, as reported by Allen et al. [5], have much lower threshold powers but have not yet been operated sufficiently far above threshold to deliver the average power desired. In addition to efficiency, the life of the pumping systems is severely limited. The tungsten-iodine lamps demonstrate at most several thousand hours of life, as compared to a 50000 -hour requirement. Both the light-emitting diodes and the potassium-mercury lamps have exhibited much shorter lives to date, the latter being measured in tens of hours [6].

If mode locking is used to generate the pulse stream in a PCM system [1], then additional difficulties will be encountered. Mode locking at a rate of $10^{9} \mathrm{pps}$ has not been demonstrated, and it will require either a very short laser cavity or the multiplexing of lower rate pulse streams as proposed by Kinsel and Denton [11]. The introduction of additional optical elements inside and outside the laser cavity will lower the efficiency further. Simultaneous intracavity doubling and mode locking reduces the efficiency at $0.53 \mu \mathrm{m}$ still further due to the conflicting requirements placed on the laser by these two processes [12].

Upon assessment of the various critical technologies, projections for parameter values were chosen as presented in Table II. All parameters given in parentheses are projections for the near term.

Based upon examination of the Nd:YAG situation, a reasonably ambitious development goal might be $0.2-\mathrm{W}$ $\mathrm{TEM}_{00}$ output with 0.2 percent efficiency at $0.53 \mu \mathrm{m}$, somewhat higher power and efficiency being available at $1.06 \mu \mathrm{m} . \mathrm{CO}_{2}$ lasers have operated to 30 percent efficiency at high powers. For the size of laser which delivers one to several watts, 10 percent is readily achieved. The choice of 3 percent efficiency for the $\mathrm{CO}_{2}$ laser was made to be consistent with the use of an internal modulator.

Modulators for visible $1 \mathrm{Gbit} / \mathrm{s}$ PCM communications systems have been demonstrated [13]. However, modifications to handle the transmitted optical power anticipated for space systems will be required. Nevertheless, modulation at 0.53 and $1.06 \mu \mathrm{m}$ appears achievable with prime power drains of the order of $10 \mathrm{~W}$. To reflect this, a choice of $P_{\text {mod }} / P_{\text {laser }}$ of 0.1 has been made (Table II). Since modulator drive power scales as $\lambda^{3}$ when including diffraction and mode filling effects [14], special techniques must be employed to realize manageable drive powers at $10.6 \mu \mathrm{m}$. Goodwin et al. [3] have shown that through the use of internal coupling modulation, fully modulated signals of appropriate power levels can be achieved with drive powers comparable to the laser prime power. This is reflected in the choice of $P_{\text {mod }} / P_{\text {laser }}=1.0$ in Table II.

Commercially available photoemitting surfaces operating in the reflection mode can exhibit 30 percent quantum efficiency at visible wavelengths. The new compound semiconductor photoemitters currently in advanced
TABLE II

Technology Projections

\begin{tabular}{llll}
\hline & \multicolumn{1}{c}{$0.53 \mu \mathrm{m}$} & \multicolumn{1}{c}{$1.06 \mu \mathrm{m}$} & $10.6 \mu \mathrm{m}$ \\
\hline System & AM (exterior) & AM (exterior) & DSSC (interior) \\
$m$ & 0.5 & 0.5 & 1.0 \\
$P_{\text {laser }}$ & $(0.2 \mathrm{~W})$ & $(1.0 \mathrm{~W})$ & $3 \mathrm{~W}$ \\
$\eta_{\text {laser }}$ & $(0.2$ percent) & $(1$ percent) & 3 percent \\
$P_{\text {mod }} / P_{\text {laser }}$ & 0.1 & 0.1 & 1.0 \\
$\eta_{T}$ & $4.5 \times 10^{-4}$ & $2.3 \times 10^{-3}$ & $1.5 \times 10^{-2}$ \\
$\eta_{\text {det }}$ & 30 percent & $(2$ percent) & 30 percent \\
$f$ & 0.5 & 0.5 & 1.0 \\
$\eta_{R}$ & 0.15 & 0.01 & 0.3 \\
\hline
\end{tabular}

Projections for near future are given in parentheses.

TABLE III

Comparison of Srgnal-to-Norse Ratros With EquaL $P_{\text {prime }}, B, R, L, A_{R}$ For All Systems

\begin{tabular}{|c|c|c|}
\hline & $\begin{array}{c}\text { Case I } \\
\text { Equal Transmitter } \\
\text { Pointing Burden }\end{array}$ & $\begin{array}{c}\text { Case II } \\
\text { Equal Optics Size } \\
\text { Burden }\end{array}$ \\
\hline Equate & $\theta_{T}$ & $D_{T}$ \\
\hline$S / N$ & $\eta_{T} \eta_{R} \lambda$ & $\eta_{T} \eta_{R} \lambda^{-1}$ \\
\hline$\frac{(\mathrm{S} / N)_{\mathrm{CO}_{2}}}{(\mathrm{~S} / N)_{2 x \mathrm{YAG}}}$ & 1333 & 3.3 \\
\hline$\frac{(S / N)_{\mathrm{CO}_{2}}}{(S / N)_{\mathrm{YAG}}}$ & 2000 & 20 \\
\hline
\end{tabular}

development are expected to deliver 2 percent at $1.06 \mu \mathrm{m}$. Photovoltaic $\mathrm{HgCdTe}$ detectors have been operated at 30 percent efficiency with $1 \mathrm{GHz}$ of bandwidth [15]. These developments serve as the basis for the parameters chosen for detector status in Table II.

The choice of a modulation index listed in Table II is somewhat arbitrary for the $10.6-\mu \mathrm{m}$ system, since it employs a suppressed carrier modulation format. The choice of unity for $10.6 \mu \mathrm{m}$ represents a conservative position and at the same time avoids the need for a much more detailed treatment. It should be recognized, however, that in-phase carrier insertion is required in the optical receiver to demodulate double-sideband suppressed carriers, and that this may not be an easy task.

To effect the system comparison, all parameters in (1) that are essentially the same for all approaches are set equal: prime transmitter power, bandwidth, range, optical loss, and receiving aperture area. The latter is the most arbitrary assumption and is made on the basis that a diffraction-limited aperture at $10.6 \mu \mathrm{m}$ and a photon bucket at $0.53 \mu \mathrm{m}$ will have similar surface figure requirements and fields of view.

The remaining dimensional variable is that of the diameter of the transmitter aperture. Two choices are made, as illustrated in Table III.

Case I: If it is assumed that pointing accuracy is the governing system burden, then an appropriate choice is to equate the transmitter beamwidths $\theta_{T}$ of (1). The relative signal-to-noise ratio is then proportional to the 


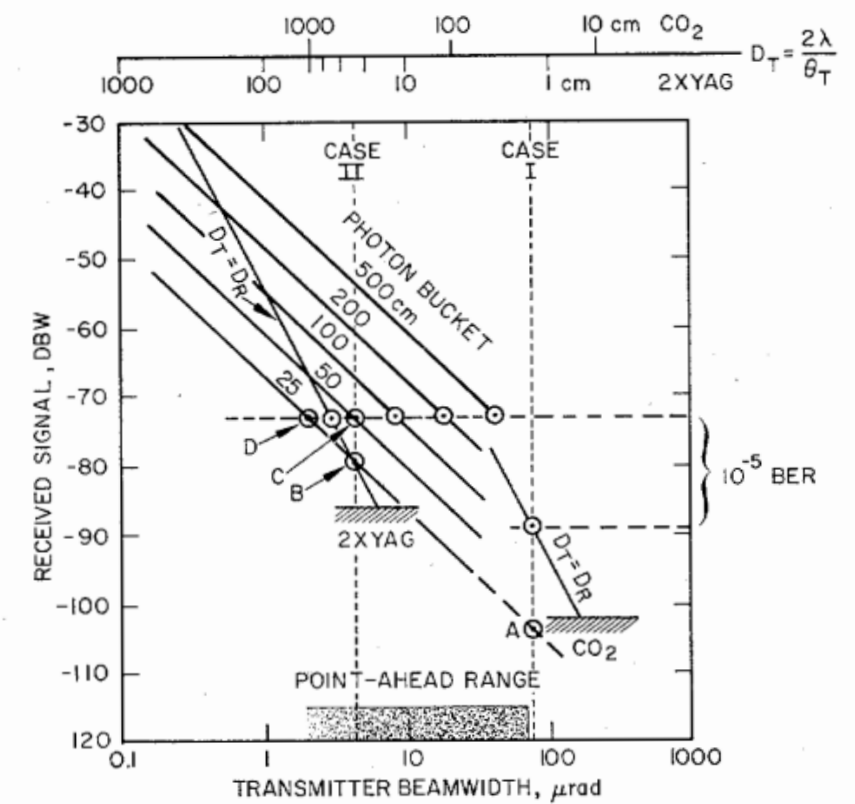

Fig. 3. Received signal as a function of transmitter beamwidth for the system parameters listed in Table II. For the Nd:YAG system, curves are shown for equal transmitter and receiver apertures $\left(D_{T}=D_{R}\right)$ and for fixed receiver apertures of varying sizes (photon bucket). Scales converting beamwidth to aperture size are given at the top of the figure. The shaded region along the abcissa gives the range of point-ahead angles encountered.

product of the remaining variables $\eta_{T} \eta_{R} \lambda$. The parameters from Table II are substituted and the results expressed as $S / N$ ratios for the three systems. The $\mathrm{CO}_{2}$ system is clearly superior when compared on this basis. It is important to emphasize that this comparison attempts to equate the pointing and tracking accuracy as the burden on the system. Therefore, this comparison is most valid for optics sizes that are dimensionally insignificant compared to the spacecraft dimension and easily managed.

Case II: The alternative spacecraft burden assumption would be to reason that pointing and tracking precision is relatively inexpensive (in terms of burden), and that the weight burden of the optics would dominate. Also, to be consistent, one must assume that the $20: 1$ ratio of surface figure control at the extreme wavelengths does not significantly affect weight. Under these circumstances, the transmitter aperture size $D_{T}$ is equated for all systems, and the results are given in the second column. Under this assumption, the signal-to-noise expression involves the remaining variables as the product $\eta_{T} \eta_{R} \lambda^{-1}$. Taking ratios as before, the relative advantages change drastically. Again, it is important to realize that the Nd:YAG systems must achieve much more precise pointing and tracking and also accommodate the complication of point-ahead under these circumstances. Case II imposes an unrealistic burden on the Nd:YAG transmitter pointing accuracy through the forced choice of a very narrow beamwidth. This illustrates the weakness of this type of comparison; it essentially ignores the practical limits on some of the parameters.

There are fundamental physical reasons for the consistent dominance of the $\mathrm{CO}_{2}$ system in this analysis. The principal factors contributing to this advantage are the lower quantum noise limits and the higher laser efficiency. Each of these contributes more than an order of magnitude advantage for $\mathrm{CO}_{2}$, despite the fact that a technology projection of about a factor of 10 for Nd:YAG efficiency has been included. Because of the consistent superiority of the $0.53-\mu \mathrm{m}$ approach over the 1.06- $\mu \mathrm{m}$ approach, only the $0.53-\mu \mathrm{m}$ system will be treated throughout the remainder of the discussion.

In an attempt to illustrate the physical significance of the assumptions for the two cases, reference is made to Fig. 3. Here the received signal is plotted as a function of transmitter beamwidth with the same parameters as given in Table II. Since absolute signal strengths are desired, an information (and noise) bandwidth of $1 \mathrm{GHz}$ is assumed. The actual bandwidth necessary for a 1-Gbit/s data rate depends on the modulation format; for simplicity we will assume here that a 1-Gbit/s data rate can be transmitted in a 1-GHz bandwidth. The transmitter aperture dimensions corresponding to the beamwidths for 10.6 and $0.53 \mu \mathrm{m}$ are shown at the top of the figure. The curves $D_{T}=D_{R}$ are for systems which employ equal transmitting and receiving apertures, while those labeled "photon bucket" assume a constant area receiving aperture with the transmitter aperture varied according to the beamwidth. The shaded planes labeled $2 x \mathrm{YAG}$ and $\mathrm{CO}_{2}$ represent the effective threshold of sensitivity for the receivers.

The horizontal dotted lines indicate the received signal levels necessary to yield $10^{-5} \mathrm{BER}$. As mentioned earlier, the BER cannot be directly related to the signal-to-noise ratio for some cases, e.g., direct photon detection. A suitable approximation is obtained by noting that, in the absence of background or detector dark current, about 11 photoelectrons per bit received will yield a $10^{-5} \mathrm{BER}$ in a direct detection system (see, e.g., $[16$, ch. 11]). To simplify the discussion, the background radiation is ignored in this comparison, even though large aperture photon buckets may collect a significant background radiation. This will be especially true for the receiver in synchronous orbit looking at a LEO satellite against a bright earth. Backgrounds greater than a fraction of a photon per bit will require stronger signals to maintain a BER of $10^{-5}$. One background photoelectron per bit adds about $3 \mathrm{~dB}$ to the signal required for $10^{-5} \mathrm{BER}$.

If the further assumption is made that the quantum noise associated with the signal amounts to 1 photoelectron per bit, then a $10^{-5}$ BER requires about $10 \mathrm{~dB}$ $S / N$ for direct detection in the absence of background. An exact equivalence is found for the heterodyne case where Gaussian statistics apply; a $10^{-5}$ BER requires $13 \mathrm{~dB}$ $S / N$ [17]. This latter value is used to define both $10^{-5}$ BER lines shown in Fig. 3, which allows for a small background in the direct detection case. Background can be completely ignored for heterodyne reception.

The lines $D_{T}=D_{R}$ give the signal levels received as a function of beamwidth when the transmitter and receiver apertures are equal, $\theta_{T}=\theta_{R}$; in this case the received 
signal varies as $\theta_{T}{ }^{-4}$. The lines labeled "photon bucket" apply only to the spatially incoherent $0.53-\mu \mathrm{m}$ system and give the received signal as a function of transmitter beamwidth for receiving apertures of the diameters indicated; in this case the received signal varies as $\theta_{T}{ }^{-2}$. Where these lines cross the $D_{T}=D_{R}$ line, the photon bucket diameter equals the transmitter aperture diameter. Note that photon buckets cannot be used with the $\mathrm{CO}_{2}$ system, since heterodyne detection requires that the received phase fronts be preserved spatially.

For the case of equal transmitter beamwidths (Case I), a $\mathrm{CO}_{2}$ laser system achieves a BER of $10^{-5}$ with $25-\mathrm{cm}$ transmitting and receiving apertures, or beamwidths of about $90 \mu \mathrm{rad}$. The $0.53-\mu \mathrm{m}$ system cannot achieve $10^{-5}$ BER at $90 \mu \mathrm{rad}$ pointing accuracy unless a receiving aperture (photon bucket) larger than $500 \mathrm{~cm}$ in diameter is used. Recalling that $D_{R}$ is assumed equal for both systems in this comparison, it is noted that the $0.53-\mu \mathrm{m}$ signal is below the quantum noise limits for a $25-\mathrm{cm}$ diameter photon bucket (point $A$ ).

To understand Case II for which the transmitter aperture diameters are chosen equal, the operating point for the $0.53-\mu \mathrm{m}$ system must move up and to the left (from point $A$ ) along the 25 -cm photon bucket curve to the point where the $D_{T}$ scale shows $25 \mathrm{~cm}$ (point $B$ ). Note that point $B$ lies below the $10^{-5}$ BER line. This indicates that the $0.53-\mu \mathrm{m}$ system cannot operate with this combination of transmitter gain and receiving aperture area (or equivalently, with this $D_{T} D_{R}$ product). To achieve the requisite signal level, either the receiving aperture diameter must be increased (e.g., to $50 \mathrm{~cm}$ as illustrated by point $C$ ) or the transmitter aperture diameter increased (e.g., to $50 \mathrm{~cm}$ as illustrated by point $D$ ). Both of these operating points involve the use of beamwidths of a few microradians, which in turn implies a pointing accuracy of a small fraction of a microradian, an extremely small value. The engineering trade to relieve this parameter obviously involves choice of a larger photon bucket and a smaller transmitter aperture, but the latitude one has in proceeding in this direction is limited, as is evidenced by examination of Fig. 3. What is obviously missing from the argument is an optimizing criterion. This criterion is developed from engineering and total burden considerations, and is the basis for the comparison to be developed in the next section.

\section{Weight-Cost Equivalence Comparison}

As described previously, the optimum system design will be that which minimizes some measure of total burden for a given channel capacity. For a satellite system, this burden is sensibly described in terms of weight. Moreover, since satellite payload in orbit can be conveniently related to launch costs, the weight burden is essentially equivalent to cost burden. Obviously, limiting the cost discussion to launch costs assumes that development costs and costs of fabricating flight hardware will be essentially comparable for the various candidates. This is true within the limits of vision at this time. The assumption obviously also becomes more valid as the number of systems launched increases, and in that sense this basis for comparison is more valid for the long term, approaching a life-cycle cost concept in the limit.

The weight contributions can be separated into three classes; 1) those associated with power generation, 2) those associated with heat rejection, and 3) the weight of the equipment itself. Based upon assessments of a variety of spacecraft engineering designs, nominal unit penalties can be established for the first two. Values of $0.11 \mathrm{lb} / \mathrm{W}$ for power generation (5-year mission) and $0.1 \mathrm{lb} / \mathrm{W}$ for heat rejection (at $300^{\circ} \mathrm{K}$ ) are used here. Equipment weights must be assessed more or less on an individual basis.

One of the key equipment items from a weight standpoint is the optical system. For this reason an attempt has been made to provide an empirical scaling law for spaceborne steered apertures with associated backups, gimbals, electronics, etc., i.e., the pointing and tracking subsystem. Several existing spacecraft have been studied and the weights of this portion of the systems assessed. All contain steered apertures one foot in diameter or less. It was found that a scaling law of the form $W=C_{1}+C_{2} D^{3}$ fits the data very well and comes reasonably close to the weight projection for a detailed paper design at 25 inch in diameter which had been carried out in another analysis. In general, it is found that mirror weights alone vary approximately as $D^{3}$, so the dependence is also qualitatively satisfying. This information together with the assumptions for the empirical curve are shown in Fig. 4. The other information on this figure will be developed later.

The weight-cost equivalence alluded to earlier is presented in Fig. 5. Based upon these data, nominal costs of $\$ 10000 / \mathrm{lb}$ for synchronous orbit and $\$ 2000 / \mathrm{lb}$ for low-altitude orbits were adopted for making comparisons.

Before system designs can be evolved for comparison purposes, a strategy must be developed which optimizes each system. It is proposed that this strategy must contain two steps. First, one must ascertain from cursory calculations, or equivalently examination of Fig. 3, whether closed-loop pointing and tracking is feasible, i.e., whether transmitter beamwidths can be greater than the maximum lead angle $(70 \mu \mathrm{rad})$. If so, a prudent design would adjust the transmitter beam accordingly in the belief that elimination of the point-ahead problem is a very attractive design feature. If a much smaller transmitter beamwidth is required, it should be narrowed as much as possible, consistent with a measure of assurance that such pointing accuracy can be achieved. It is obvious from previous discussions that the $\mathrm{CO}_{2}$ system is typical of the former case, and that the Nd:YAG systems are typical in the latter category.

Once these decisions have been made, the remaining degrees of design freedom are evident from an examination of (1). It turns out that the product of the parameters $P_{L}, A_{T}$, and $A_{R}$ must be maximized, where $A_{T}$ is the transmitter aperture area (proportional to $D_{T}{ }^{2}$ ). $P_{L}$ is 


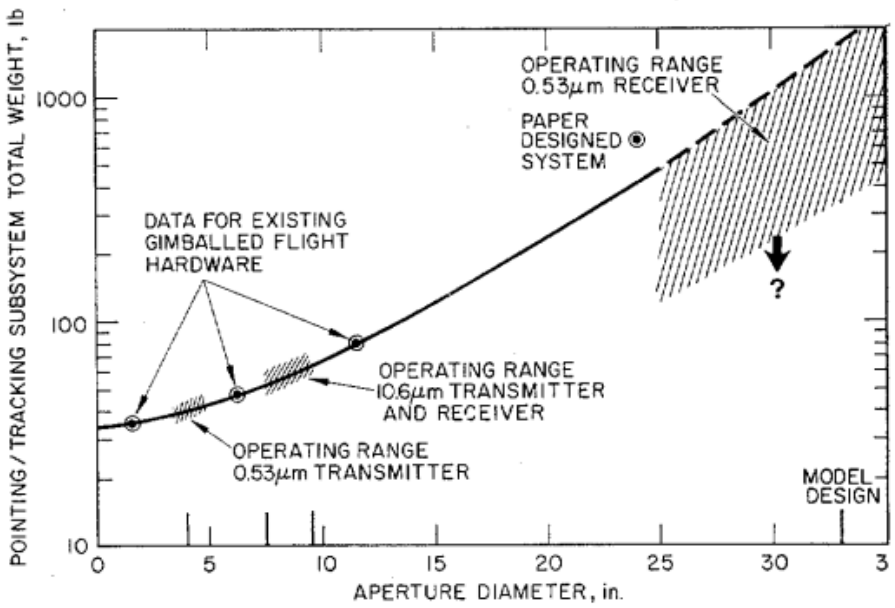

Fig. 4. Weight of pointing and tracking subsystem as a function of optical aperture diameter. The assumptions used in obtaining this empirical curve are 1) light-weight mirror technology is used (i.e., beryllium), 2) mirror figure is controlled to about $1 \mu \mathrm{m}$, 3 ) gimbaled coverage larger than a hemisphere, and 4) pointing capability of the order of 10 to $100 \mu \mathrm{rad}$.

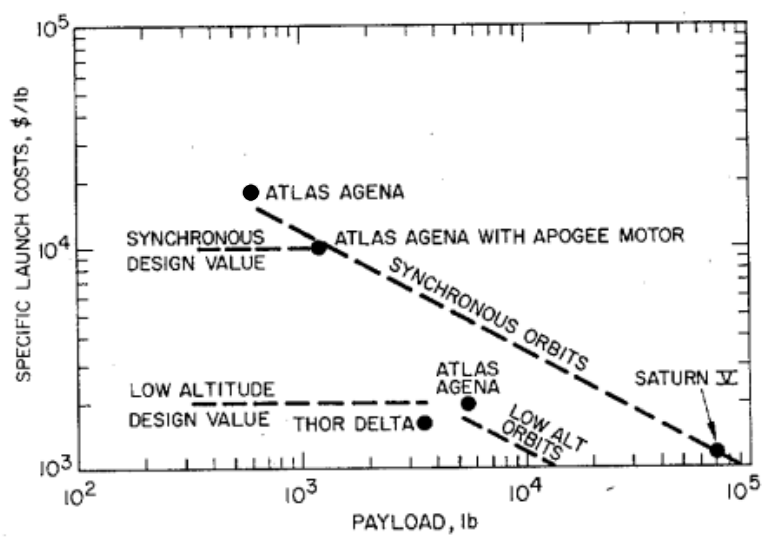

Fig. 5. Payload launch cost as a function of overall payload weight for both synchronous altitude (upper curve) and LEO altitude (lower curve). The various vehicles that would be used for the different payload sizes are shown as points along the curve.

chosen at its maximum practical value, since its associated weight burden is essentially linearly dependent. Next, $A_{T}$ should be chosen about equal to $A_{R}$ to minimize the total weight burden if $A_{T}$ yields a practical (i.e., not too small) transmitter beamwidth. If it does not, $A_{T}$ is chosen smaller than $A_{R}$ to yield the desired transmitter beamwidth limited by the pointing and tracking capability. When this strategy is followed, it turns out that both approaches require a smaller transmitter aperture; however, for the $\mathrm{CO}_{2}$ system the difference is nominal, while for the Nd:YAG system it is drastic.

The final step in the optimum design procedure involves consideration of all other burdens, including acquisition beacons, track beacons, transmitter weights, etc., for second-order modification to weight distributions and for contributions to total burden.

By following this design philosophy, the system designs which are summarized in Tables IV and V for $0.53 \mu \mathrm{m}$ and $10.6 \mu \mathrm{m}$ were evolved. In order to make the designs equivalent, a signal-to-noise ratio of $13 \mathrm{~dB}$ was chosen, as before, to give $10^{-5} \mathrm{BER}$. An information bandwidth of $1 \mathrm{GHz}$ is assumed for both systems. Optical train losses of $3 \mathrm{~dB}$ each are allowed for transmitter and receiver. The laser power was chosen to be compatible in both cases with the supply of $110 \mathrm{~W}$ of prime power to the transmitter and also consistent with the technology projections of Table II.

With these choices and the other parameter assumptions for the system as shown, the products of the aperture diameters required for the two systems are $D_{T} D_{R}=$ $8.5 \times 10^{-2} \mathrm{~m}^{2}$ for $0.53 \mu \mathrm{m}$, and $4.67 \times 10^{-2} \mathrm{~m}^{2}$ for $10.6 \mu \mathrm{m}$. Choosing the minimum transmitter beamwidth which can be comfortably accommodated as $10 \mu \mathrm{rad}$ (an estimate of practical technology projections ${ }^{1}$ based on a pointing accuracy limit of the order of $1 \mu \mathrm{rad}$ ) for the $0.53-\mu \mathrm{m}$ system, the transmitter aperture size is $10 \mathrm{~cm}$. To achieve the required signal-to-noise ratio, the receiving aperture diameter must be $85 \mathrm{~cm}$.

This dimension presents the most critical judgement problem in making the comparison. With reference to Fig. 4, it is noted that pointing and tracking subsystems with optics of this size can be expected to weigh close to $1000 \mathrm{lb}$. However, two of the assumptions which are made in generating this empirical curve do not necessarily hold for the photon bucket in this model system. First, figure control to $1 \mu \mathrm{m}$ is not needed, and second, since the photon bucket is on the synchronous satellite, hemispherical coverage is not required. The area shown shaded in Fig. 4 for the $0.53-\mu \mathrm{m}$ receiver is represented as an area of uncertainty about this subsystem, and the question mark at about $100 \mathrm{lb}$ represents a very ambitious estimate of the minimum weight which might be achieved. In any event, a range of 100 to $1000 \mathrm{lb}$ is believed to be a reasonable estimate for this subsystem.

The other data presented in Table IV are best engineering estimates. The reduction to a total equivalent weight and launch cost is self-evident from examination of the summary data. The asterisks in the choices of weight and cost associated with $D_{R}$ mean that the 100-lb number was used in computing the cost figure for want of a better criterion.

In the $10.6-\mu \mathrm{m}$ system, the transmitter beamwidth was chosen to be $100 \mu \mathrm{rad}$ in order to be significantly larger than the maximum lead angle. This implies a diameter of $19.2 \mathrm{~cm}$ for the transmitter aperture and $24 \mathrm{~cm}$ for the receiving aperture. An examination of Fig. 4 shows that these are well within the design experience range and that the pointing and tracking subsystem weight can be predicted with considerable assurance. The remaining

\footnotetext{
1 The precision required in these pointing systems cannot be achieved by passive structural control of optical axis of telescopes and associated mirror trains. Dynamic boresight control is required, and this requires the use of a reference axis. One possibility for this reference is the line of sight to the other terminal as determined by a tracking sensor. This approach appears attractive for use with common or coaxial optics (for transmit and receive) and closedloop tracking and pointing. It does not appear to resolve the dilemma in establishing the optical axis for transmission in a predictive or point-ahead system.
} 
TABLE IV

$0.53-\mu$ M System Design Summary

\begin{tabular}{|c|c|c|c|c|c|}
\hline \multicolumn{6}{|l|}{ Wideband $T_{x}$ station } \\
\hline Transmitter optics ${ }^{\mathbf{b}}$ & 33 & 30 & 30 & 39.3 & 78 \\
\hline Other components & 13 & 50 & 50 & 23.5 & 47 \\
\hline Totals & 50 & 190 & 189.8 & 89.8 & 179 \\
\hline \multicolumn{6}{|l|}{$\begin{array}{l}\text { Wideband } R_{x} \text { station } \\
\text { Receiver }\end{array}$} \\
\hline Receiver optics ${ }^{\mathrm{d}}$ & $100^{e}$ to & & & & \\
\hline & 1000 & 50 & 50 & $110.5^{\mathrm{e}}$ & $>1100^{\mathrm{e}}$ \\
\hline Tracking beacon & 4 & 90 & 89 & 23 & 230 \\
\hline Acquisition beacon & 10 & 2 & 2 & 10.5 & 105 \\
\hline Other components & 30 & $5 \overline{5}$ & $5 \overline{5}$ & 41.1 & 410 \\
\hline Totals & 144 & 197 & 196 & 185.1 & $>1845$ \\
\hline
\end{tabular}

a Assumes $P_{\text {nger }}=0.2 \mathrm{~W}, m=0.5, \eta_{T}=4.54 \times 10^{-4}, P=110 \mathrm{~W}$.

Assumes $D_{T}=10 \mathrm{~cm}, \theta_{T}=10 \mu \mathrm{rad}$.

- Assumes $f=0.5, \eta_{D}=0.3$.

d Assumes $D_{R}=85 \mathrm{~cm}$.

TABLE $\mathrm{V}$

10.6- $\mu$ M System Design Summary

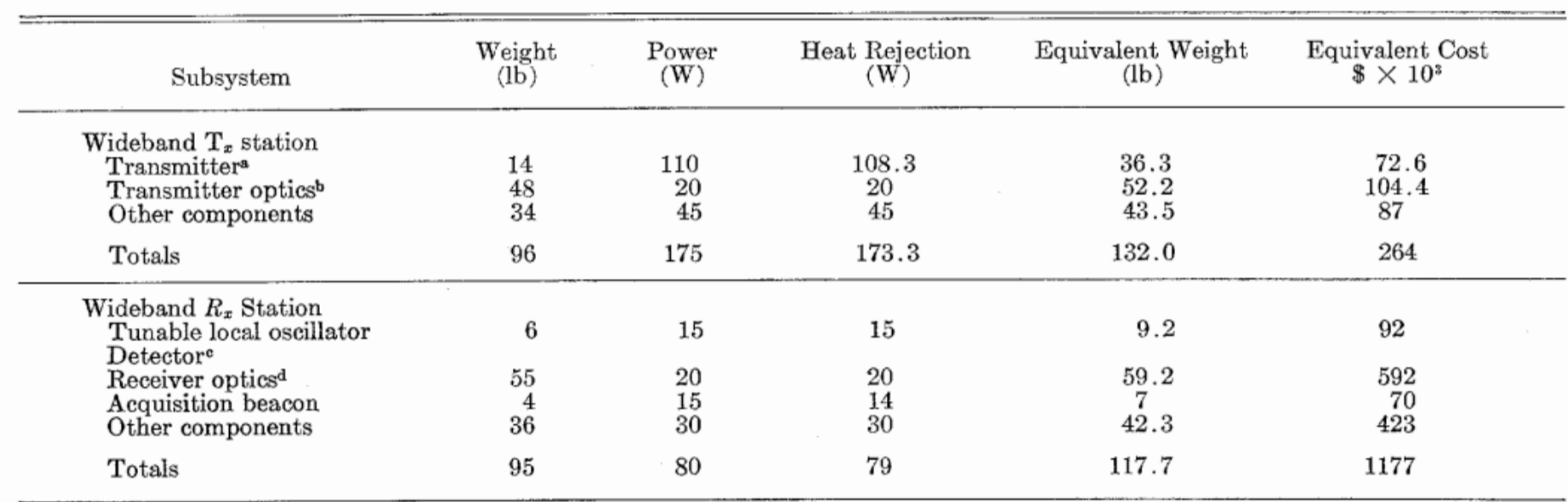

s Assumes $P_{\text {laser }}=1.65 \mathrm{~W}, m=1.0, \eta_{T}=2.5 \times 10^{-2}, P=110 \mathrm{~W}$.

b Assumes $D_{T}=19.2 \mathrm{~cm}, \theta_{T}=100 \mu \mathrm{rad}$.

c Assumes $f=1.0, \eta_{D}=0.3$.

d Assumes $D_{R}=24 \mathrm{~cm}$.

TABLE VI

Summary Comparison

\begin{tabular}{lcc}
\hline \hline & $0.53 \mu \mathrm{m}$ & $10.6 \mu \mathrm{m}$ \\
\hline$T_{x}$ weight burden (lb) & 90 & 132 \\
$R_{x}$ weight burden $(\mathrm{lb})$ & $184^{\mathrm{a}}$ & 111 \\
$T_{x}$ launch cost $\left(\$ \times 10^{3}\right)$ & 180 & 264 \\
$R_{x}$ launch cost $\left(\$ \times 10^{3}\right)$ & $>1842^{\mathrm{a}}$ & 1170 \\
\hline
\end{tabular}

$T_{x}$ on low earth orbiting satellite. $R_{x}$ on synchronous satellite.

a Assumes 100-1b photon bucket. 
data in Table $\mathrm{V}$ are again based upon engineering estimates, and the total weight and cost burdens computed as before.

The burdens for both systems are summarized in Table VI. The asterisk is retained to reflect the uncertainty introduced by the lack of information on large, steerable photon buckets suitable for space, and the fact that an optimistic value was chosen for that burden.

An appraisal of these numbers would indicate that, while there is a cost difference apparent, it is not sufficiently great to specify one or the other system. This judgment is contingent upon the fact that the numbers are based upon estimates which, of necessity, are somewhat soft, and on the observation that at least several transmitter systems may be launched for each receiver system. This does not mean that both approaches have comparable prospects for success at this point in time. Rather, the final comparison and judgement must be made upon assessment of the probability of achieving the increments in technical performance which have been projected for each candidate system. This will be the subject of discussion in the next section.

\section{Critical Technology Assessment}

Fig. 6 is included as a guide in identifying and comparing the specific technology deficiencies for each approach. The deficiencies which are deemed most serious are shown shaded; the others are areas requiring more effort, but a question of feasibility is not believed to exist. As indicated, the performance of solid-state drivers at $1 \mathrm{Gbit} / \mathrm{s}$ when operating with optical modulators (which require high peak voltages) is substantially below that which is desired. This is particularly true for the baseband systems considered here.

In general, the deficient areas are essentially the same for the Nd:YAG systems at both 0.53 and $1.06 \mu \mathrm{m}$. The only differences relate to questions about doubler crystal damage for $0.53-\mu \mathrm{m}$ operation and the reliability and life of the compound semiconductor photoemitter detectors at $1.06 \mu \mathrm{m}$.

As illustrated in Fig. 2, the desired power-efficiency combination for the Nd:YAG laser has not been achieved by approximately an order of magnitude. Even assuming the successful realization of this goal, there is a substantial question of the lifetime of the most promising pump sources (tungsten-iodine lamps and light-emitting diodes).

Problems relating to mode-locking Nd:YAG lasers mentioned in Section III have been omitted from Fig. 6, since this method of PCM generation is only one alternative (for example, compare [1] and [2]); however, it should be considered a technology deficiency for systems that employ it.

Pointing with an accuracy of the order of $1 \mu \mathrm{rad}$ (that is, a fraction of the $10-\mu \mathrm{rad}$ beamwidth) is required for the Nd:YAG systems; although a severe challenge it is primarily an engineering rather than a fundamental problem. Achieving this pointing accuracy with open-loop

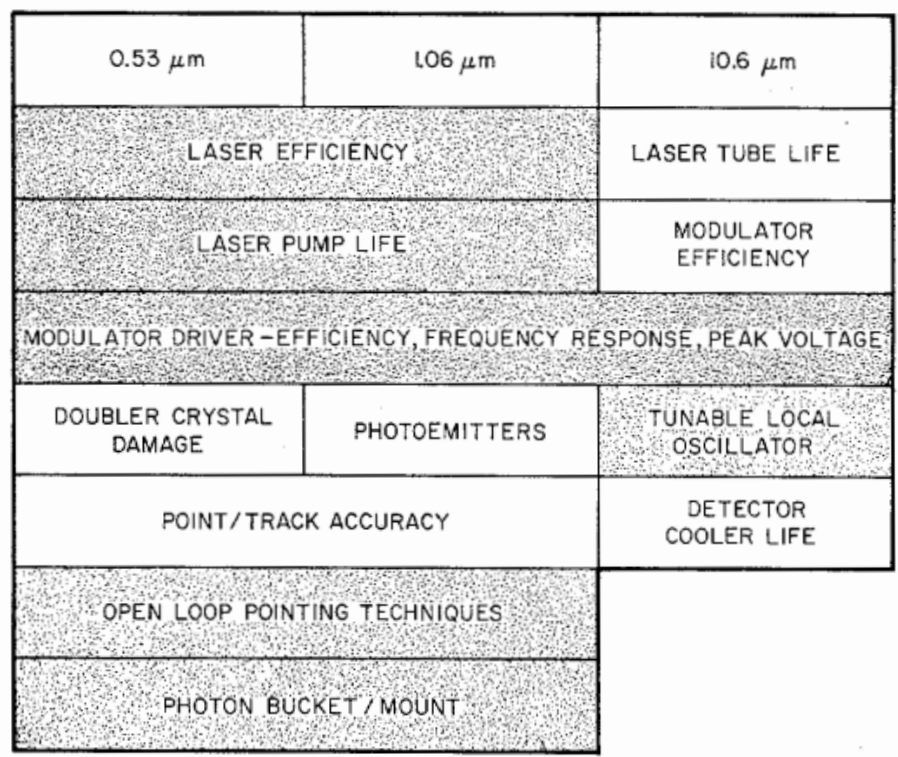

Fig. 6. Critical technology areas for the three laser systems considered. Shaded blocks indicate those areas where the technology is deficient at present.

tracking and point-ahead features (i.e., predictive pointing to $70 \mu \mathrm{rad}$ ) is believed to be a much more demanding task. Finally, the design of a lightweight photon bucket, its structural backing and associated gimbals, etc., is a challenging problem. As indicated in this study, if subsystems employing apertures approaching $1 \mathrm{~m}$ in diameter cannot be developed with weights in the range of $100 \mathrm{lb}$, the weight penalty incurred could prove to be a serious competitive disadvantage.

For the 10.6- $\mu \mathrm{m}$ system, the modulator driver is a more severe problem than for the shorter wavelength, because more drive power is required. The intracavity modulation technique described by Goodwin et al. [3] indicates the present preferred direction. The other critical problem is the realization of an adequate tunable $10.6-\mu \mathrm{m}$ local oscillator or equivalent technique that can track continuously over the anticipated Doppler shifts of \pm 700 $\mathrm{MHz}$, and, particularly, do so without garbling the information as the Doppler shift passes through zero. Although several possible solutions are envisioned, none have been demonstrated to date. Of lesser concern is the laser tube life. Although it is doubtful that a single fill of the chemically active gases can be expected to provide 50000 hours of life, it is relatively straightforward to obtain several thousand hours of life and to provide means to periodically replenish or stabilize the gas by a variety of methods. Modulator efficiency is of concern primarily because of the burden it places upon the driver, and because this component consumes an amount of prime power comparable to the laser itself. Finally, although radiation coolers for the mercury cadmium telluride detectors exist and appear to work in the laboratory, they have yet to be proven in space. The $\mathrm{CO}_{2}$ system has the advantage that the aperture sizes at both terminals are relatively small ( $\lesssim 25 \mathrm{~cm}$ diameter), the beamwidths are 
sufficiently broad that closed-loop tracking and pointing can be employed, and that tracking and pointing accuracies are relaxed by an order of magnitude below those for the Nd: YAG system.

\section{Conclusions}

In the quest for 1-Gbit/s space relay links, it is certainly too early to preempt options to choose either a $0.53-\mu \mathrm{m}$ $\mathrm{Nd}$ :YAG or a $10.6-\mu \mathrm{m} \mathrm{CO}_{2}$ system approach because both require performance increments and innovations. Comparisons from a fundamental point of view indicate that the $10.6-\mu \mathrm{m}$ system has an advantage in signal-to-noise ratio. This advantage derives naturally from the longer wavelength, and thus lower quantum noise limits, and the exceptional efficiency of the $\mathrm{CO}_{2}$ laser. To achieve near-quantum noise-limited performance, however, the additional complexity of heterodyne detection and Doppler tracking must be accommodated. The direct detection receiver concept for the $\mathrm{Nd}$ :YAG system is simpler by comparison. On the other hand, to compensate for the fundamental advantages of the 10.6- $\mu \mathrm{m}$ approach, very narrow transmitter beams must be employed, which introduce challenging pointing and tracking problems including point-ahead, and very large receiving apertures must be used. The choice between the approaches thus reduces to an assessment of the potential for solving the remaining deficiencies in technology for each. For the $\mathrm{Nd}$ : YAG or any visible system, laser power and efficiency are the crucial problems. Only by increasing efficiency above 0.2 percent can some relief be provided for the problems anticipated in the use of large receiving apertures and very narrow beams. For the $\mathrm{CO}_{2}$ system, concentration on the problem of Doppler shift compensation is mandatory. Once this is solved, there appear to be no other severe questions of a fundamental nature for this approach.

\section{REFERENCES}

[1] M. Ross, S. I. Green, and J. Brand, "Short-pulse optical communications experiments," Proc. IEEE, vol. 58, pp. 1719-1726, Oct. 1970.

[2] P. S. Castro, K. K. Chow, H. V. Hance, W. B. Leonard, R. C. Ohlmann, S. E. Patterson, D. G. Peterson, R. B. Ward, and R. F. Whitmer, "A 1-GBit/see CW laser communication experiment," presented at the 1971 IEEE/OSA Conf. on Laser Engineering and Applications, Washington, D. C., June $2-4,1971$.

[3] J. E. Kiefer, T. A. Nussmeier, and F. E. Goodwin, "Intracavity CdTe modulators for $\mathrm{CO}_{2}$ lasers," this issue, pp. 173-180.

[4] R. B. Allen and S. J. Scalise, "Continuous operation of a YAlG:Nd laser by injection luminescent pumping," Appl. Phys. Lett., vol. 14, pp. 188-190, Mar. 15, 1969.

[5] R. B. Allen, E. G. Dierschke, B. C. Gilbert, and R. W. Haisty "Room-temperature operation of a diode-pumped $\mathrm{YAG}$ : Nd laser," IEEE J. Quantum Electron. (Abstracts), vol. QE-7, pp. 300-301, June 1971.

[6] I. Liberman, D. A. Larson, and C. H. Chureh, "Efficient $\mathrm{N} d$ :YAG CW lasers using alkali additive lamps," IEEE $J$. Quantum Electron., vol. QE-5, pp. 238-241, May 1969.

[7] J.D. Foster and L. M. Osterink, "Thermal effects in a Nd:YAG laser," J. Appl. Phys., vol. 41, pp. 3656-3663, Aug. 1970

[8] V. Evtuhov, private communication.

[9] R. B. Chesler and D. Maydan, "Short, efficient, and stable $\mathrm{Nd}$ :YAG laser cavities using imaged mirrors," IEEE J. Quantum Electron. (Abstracts), vol. QE-7, p. 298, June 1971.

[10] J. E. Geusic, W. B. Bridges, and J. I. Pankove, "Coherent optical sources for communications," Proc. IEEE, vol. 58, pp. 1419-1439, Oct. 1970.

[11] T. S. Kinsel and R. T. Denton, "Terminals for a high-speed optical pulse code modulation communication system, pt. IIoptical multiplexing and demultiplexing," Proc. IEEE, vol. 56 , pp. 146-154, Feb. 1968.

[12] C. B. Hitz and L. M. Osterink, "Simultaneous intracavity, frequency doubling and mode locking in a Nd:YAG laser," A ppl. Phys. Lett., vol. 18, pp. 378-380, May 1971.

[13] G. White, "A one-gigabit-per-second optical PCM communications system," Proc. IEEE (Lett.), vol. 58, pp. 1779-1780, Oct. 1970.

[14] I. P. Kaminow and E. H. Turner, "Electrooptic light modulators," Proc. IEEE, vol. 54, pp. 1374-1390, Oct. 1966.

[15] C. Vérié and M. Sirieix, "Gigahertz cutoff frequency capabilities of CdHgTe photovoltaic detectors at $10.6 \mu$," this issue, pp. $180-184$.

[16] W. K. Pratt, Laser Communication Systems. New York: Wiley, 1969 .

[17] J. G. Lawton, "Comparison of binary data transmission systems," in Proc. 2nd Annu. Nat. Conv. Military Electronics, 1958. 